\title{
Fisiología de la perfusión medular
}

\author{
Jorman Harvey Tejada Perdomo ${ }^{1} D^{\star}$, José D. Charry² MS*. Jaime Andrés Salcedo Cerquera ${ }^{3} \mathrm{MD}^{*}$. \\ 1 Neuroanestesiólogo, Especialista en Gerencia de Servicios de Salud. \\ Director Posgrado de anestesiología. \\ Universidad Surcolombiana. Hospital Universitario Hernando Moncaleano Perdomo. Neiva, Colombia. \\ 2 Estudiante de Medicina Universidad Surcolombiana. Hospital Universitario Hernando Moncaleano Perdomo. Neiva, Co- \\ lombia. \\ 3 Médico Residente de Anestesiología y Reanimación. \\ Universidad Surcolombiana. Hospital Universitario Hernando Moncaleano Perdomo. Neiva, Colombia. \\ * Investigadores Grupo de Desarrollo social, Salud Pública y derechos humanos. Semillero de Anestesiología.
}

Rev. Chil. Neurocirugía 42: 8-11, 2016

\section{Resumen}

Introducción: En las últimas décadas ha aumentado el interés por el estudio de la fisiología de la perfusión a nivel de la médula espinal. Muchos de los tratamientos en pacientes con lesión medular han sido basados en el paralelismo de la dinámica vascular entre el cerebro y la médula. Conocer estos aspectos así como los métodos relacionados con su monitoria resulta favorable para la adecuada intervención del paciente con lesión a nivel medular. Objetivo: Realizar una revisión en la literatura científica de los aspectos más importantes que intervienen en la perfusión de la médula espinal, los mecanismos de autorregulación y su aplicación clínica dentro del estudio de la fisiología medular. Métodos: Con las palabras claves, se realizó una revisión no sistemática en las bases de datos correspondientes a OVID, Medline/PubMed, Science Direct. Resultados y Conclusiones: El papel de la autorregulación es vital en la conservación de la integridad de la médula espinal, realizar un adecuado control de ella así como de otros factores químicos y metabólicos son determinantes en el control del flujo sanguíneo medular y minimiza el riesgo de lesión medular secundaria. Las curvas de autorregulación para el cerebro y la médula espinal son virtualmente idénticas; dentro de un rango de 60-150 $\mathrm{mmHg}$.

Palabras clave: Cordón espinal, presión de perfusión, autorregulación, flujo sanguíneo medular.

\begin{abstract}
Introduction: Over the past decades the interest in the study of the physiology of perfusion at the level of the spinal cord has increased. Many treatments in patients with spinal cord injury have been based on the parallelism of the vascular dynamics between the brain and the spinal. Knowing about these aspects and methods related to their monitoring practice is favorable for a proper intervention of the patient with spinal cord injury. Objective: To do a scientific literature review on the most important aspects involved in spinal cord perfusion, autoregulatory mechanisms and their clinical applications in the study of spinal cord physiology. Methods: Using the keywords, a non-systematic review on the OVID, Medline/PubMed and Science Direct databases, was performed. Results and Conclusion: The role of autoregulation is vital in maintaining the integrity of the spinal cord, an adequate control of it as well as other chemical and metabolic factors are important in the control of medullary blood flow and minimizes the risk of secondary spinal cord injury. The autoregulation curves for the brain and spinal cord are virtually identical, within a range of $60-150 \mathrm{mmHg}$.
\end{abstract}

Key words: Spinal cord, perfusion pressure, autoregulation, medullary blood flow. 


\section{Introducción}

Un mecanismo intrínseco del sistema cerebrovascular es mantener el flujo sanguíneo del tejido dentro de un rango estrecho a pesar de los cambios en la presión de perfusión, ésto ha sido establecido como mecanismo de regulación primario del flujo sanguíneo en el cerebro, situación que desde el punto de vista fisiológico es de mayor importancia para mantener la homeostasis en el medio interno cerebral ${ }^{1}$.

A pesar de que se han presentado avances tecnológicos a nivel de la monitoria de la perfusión cerebral, se han encontrado limitaciones en la extrapolación de estos estudios al contexto de la perfusión de la medula espinal principalmente por aspectos como la dificultad en la canalización y la tendencia al vasoespasmo de los vasos arteriales espinales, la dificultad en el aislamiento del tejido del cordón espinal y la falta de un lugar adecuado para el muestreo venoso; por la complejidad en la luz y el pequeño tamaño del sistema de drenaje venoso medular².

\section{Métodos}

Revisamos artículos en la base de datos MEDLINE a través del buscador PUBMed, usando los términos MeSH "Spinal cord" AND "perfusion pressure" AND "autoregulation" AND "blood flow"; seleccionando idioma español e inglés y por año desde 1970 hasta 2014. Excluyendo cartas al editor y reportes de caso, dando mayor relevancia a los artículos de revisión. Se incluyeron además artículos de la base de datos LILACS con los mismos criterios de búsqueda.

\section{Circulación y flujo sanguíneo medu- lar}

El patrón básico de irrigación arterial de la médula espinal involucra tres vasos que corren longitudinalmente a lo largo de ella: la arteria espinal anterior y dos arterias espinales posteriores ${ }^{3}$. La arteria espinal anterior nace de la unión de dos ramas de la arteria vertebral que se unen a nivel de la decusación de las pirámides para luego descender por la superficie anterior de la médula, un poco dentro del surco medio anterior; Irriga las pirámides medulares y las estructuras medulares paramedianas más los dos tercios anteriores de la médula espinal; el resto depende de las arterias medulares y radiculares segmentarias siendo la más importante la arteria radicular magna o de Adamkiewicz, generalmente a la altura de $\mathrm{T} 10$ a $\mathrm{L} 1^{4}$.

Las arterias espinales posteriores nacen directamente de las arterias vertebrales o indirectamente de las arterias cerebelosas postero-inferiores, para luego descender por la superficie posterolateral e irrigar el tercio posterior de la médula espinal ${ }^{5}$. El sistema longitudinal es reforzado por una serie muy variable de vasos tributarios transversales que penetran al canal medular por los agujeros intervertebrales junto a los nervios y raíces espinales ${ }^{6}$. Las anastomosis entre los vasos longitudinales y los vasos segmentarios se producen en la superficie de la médula espinal (Figura 1).

El drenaje venoso de la medula espinal se da a través de dos plexos y venas con una distribución similar al circuito arterial. El plexo vertebral interno es una red anastomótica de venas que se entremezcla con el tejido conjuntivo laxo del espacio epidural, siendo una vía de diseminación de células neoplásicas muy común hacia el cerebro. El plexo vertebral externo, formado por un conjunto de venas que corren longitudinalmente por la superficie externa de los cuerpos y láminas vertebrales ${ }^{7}$. Su porción anterior se comunica con las venas basivertebrales y la posterior con las venas occipitales, cervicales profundas y vertebrales ${ }^{6}$.

\section{Autorregulación y presión de perfu- sión medular}

La autorregulación cerebral es la habilidad de los vasos cerebrales para reaccionar a cambios en la presión arterial (PA) y en la presión de perfusión cerebral (PPC), con el fin de lograr un flujo sanguíneo cerebral estable (Figura 2). El flujo sanguíneo cerebral (FSC) es determinado por la PPC y la resistencia cerebrovascular (RV); $F S C=P P C / R V^{8}$. En general, el FSC se mantiene constante en un rango de PPC entre 60-150 $\mathrm{mmHg}$. Si la autorregulación cerebral falla, el FSC varia directamente con los cambios de PPC y el cerebro queda en riesgo de daño isquémico ante hipotensión arterial o de hemorragia ante hipertensión arterial; El FSC cambia paralelamente a los cambios en la $\mathrm{PA}^{9}$. La autorregulación ha sido ampliamente investigada en la vasculatura cerebral, considerablemente menos información hay en la autorregulación del flujo sanguíneo medular (FSM). Algunos estudios realizados en animales han demostrado niveles de FSM en la sustancia gris de $61 \mathrm{ml} / 100 \mathrm{~g} / \mathrm{min} \mathrm{y}$ en la sustancia blanca de $15 \mathrm{ml} / 100 \mathrm{~g} /$ $\min ^{10}$. Con base en éstas y otras afirmaciones ${ }^{11}$ se habla de un paralelismo entre la dinámica vascular cerebral y la vasculatura espinal siendo un punto de referencia durante el tratamiento de la lesión a nivel medular. Las curvas de autorregulación para el cerebro y la médula espinal son virtualmente idénticas dentro de un rango de 60-150 $\mathrm{mmHg}^{1}$.

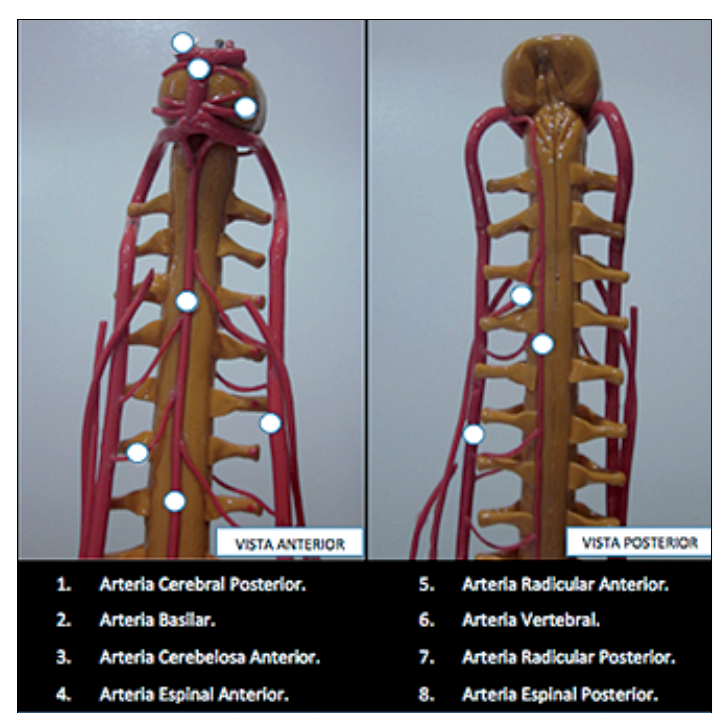

Figura 1. Anatomía arterial y venosa de la médula espinal. 


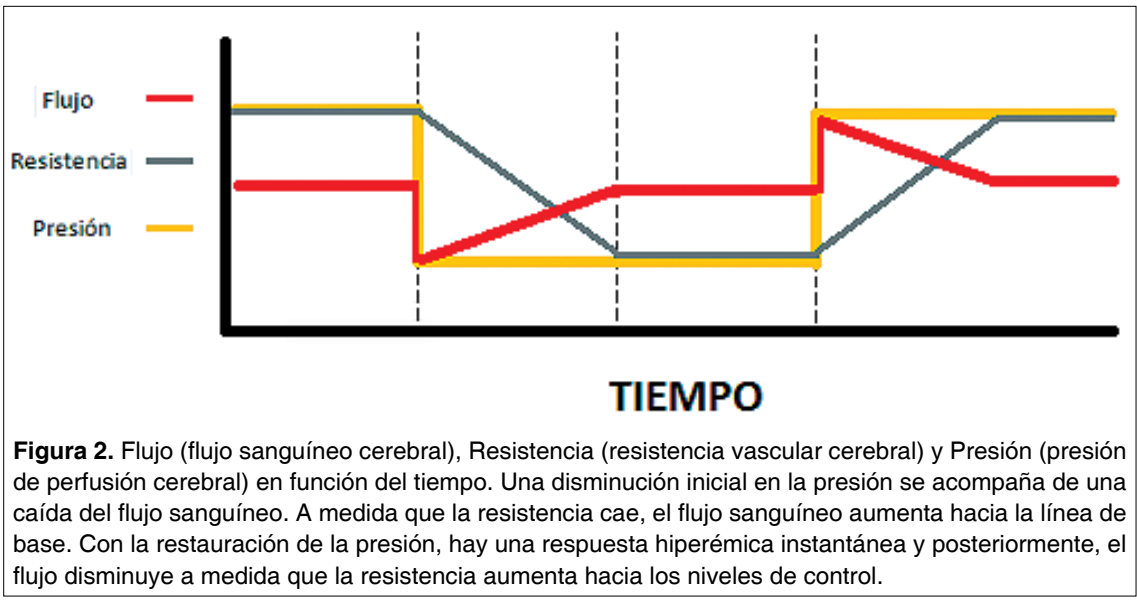

Por otro lado, es importante mencionar que dentro del abordaje del trauma cráneo encefálico severo (TCEs) se tiene estandarizado la medición de la PA invasiva y de la presión intracraneana (PIC), como reflejo de los cambios dentro de los compartimientos de la bóveda craneana, cuyo objetivo es optimizar la PPC, PPC=PAM-PIC ${ }^{12}$. Dicha intervención, al igual que las demás (normocapnia, osmóticos, vasopresores, cirugía, etc.) lo que busca es evitar la lesión secundaria. En contraste, durante la cirugía en trauma raquimedular la mayoría de grupos médicos no miden la PA invasiva, por tanto niveles óptimos de PAM y PaCO2 son desconocidos. La Asociación Americana de Neurocirujanos (AANS) concluye que hay insuficiente evidencia que soporte un tratamiento estandarizado pero recomienda mantener PAM $85-90 \mathrm{mmHg}$ por 5-7 días en la lesión traumática de la médula espinal ${ }^{13}$, mientras que en el Reino Unido recomiendan una presión sistólica 90-100 mmHg como opción de tratamiento ${ }^{14}$.

Actualmente no hay un método clínico para medir la presión intraespinal (PIE) en lesión medular, como se mide la PIC en TCEs.

Lograr monitorizar la presión de perfusión medular (PPM) siendo PPM=PAMPIE sería un avance al permitir optimizar las metas hemodinámicas y reducir la lesión secundaria ${ }^{15}$. También permitiría dilucidar el efecto de las intervenciones (cambios $\mathrm{PaCO} 2, \mathrm{PA}$, etc.) sobre la perfusión medular ${ }^{16}$.
Se ha descrito como PPM óptima 90 tracraneal, la hiperperfusión y/o la hipoperfusión se asocian con daño en la autorregulación, lo cual causa lesión medular secundaria ${ }^{17}$. Maniobras que influencian la PIC como PaCO2 o manitol, tienen poco efecto sobre la PIE y PPM; sin embargo, los inotrópicos por efecto sobre la PAM sí han demostrado un aumento neto en la $\operatorname{PPM}\left({ }^{2}\right)$.

\section{Monitoría de la perfusión medular} Las primeras medidas del flujo sanguíneo a nivel medular fueron tomadas con autoradiografía, técnica que ofrece pobre capacidad de detectar cambios causados por la administración de drogas u otras variables sobre la perfusión. Se conocen reportes bajo ésta técnica de valores de flujo sanguíneo en la médula espinal entre 10 y $20 \mathrm{ml} / 100 \mathrm{~g} /$ min para la sustancia blanca y 41 a 63 $\mathrm{ml} / 100 \mathrm{~g} / \mathrm{min}$ para la sustancia gris ${ }^{18}$.

El advenimiento del ultrasonido Doppler permitió medir directamente el flujo sanguíneo medular en cirugías que involucran pinzamiento aórtico y por ende, alteraciones a éste nivel podrían ser investigadas a través de mediciones continuas ${ }^{19}$

En los últimos años, el desarrollo de técnicas no invasivas como la Espectrofotometría Cercana al Infrarrojo (NIRS) ha permitido la evaluación y monitoria de la hemodinámia cerebral ${ }^{20}$, su uso en combinación con el marcador indocianina verde ha sido ampliamente usado como monitoria $\mathrm{mmHg}$ por analogía a la dinámica in- intraoperatoria de la perfusión medular en estudios animales ${ }^{21}$. Estos estudios han logrado detectar isquemia medular más temprano con el NIRS que con potenciales evocados motores ${ }^{22}$.

Amir y $\mathrm{Col}^{23}$., identificaron mediante mediciones realizadas con el NIRS sobre la dura y las láminas, un incremento en el FSM en respuesta a la hipercapnia. Dichos cambios en la perfusión medular son un reflejo de lo observado a nivel del FSC. La aplicación de estas técnicas podrían proporcionar una visión más amplia de la fisiopatología de la lesión y su uso quizás sería ventajoso para detectar tempranamente, o impedir la lesión medular.

Finalmente, cada vez toma más fuerza la posibilidad de realizar una monitoria continúa de la PIE tanto en casos de lesión medular traumática como en otras condiciones que causan edema de la medula espinal (ej. Mielitis transversa $)^{24}$, mediante la colocación de sondas a nivel subdural (por encima, por debajo y en el sitio de la lesión) y extradural. Dicha monitoria comprende el registro de la PA invasiva y el análisis continuo de la ondas de PIE las cuáles, al igual que las ondas de PIC; comprenden 3 picos que corresponden al pulso arterial, la compliance intracraneal y el cierre de válvula aórtica. Si el resultado del análisis es una PIE baja (< $10 \mathrm{mmHg}$ ) se considera una adecuada reserva compensatoria; con PIE 10-15 $\mathrm{mmHg}$ la reserva compensatoria está agotada y con una PIE 15-20 $\mathrm{mmHg}$ hay una pérdida progresiva de la autorregulación ${ }^{25}$.

En conclusión, conocer y evaluar el FSM es esencial en el manejo de pacientes con patologías espinales; Realizar una adecuada monitoria y control de la autorregulación, así como de los otros factores químicos y metabólicos determinantes en el flujo sanguíneo medular, permiten minimizar los riesgos de lesión medular secundaria.

Financiación: Propio de los autores.

Conflicto de intereses: Ninguno.

Recibido: 13 de agosto de 2015 Aceptado: 20 de octubre de 2015 


\section{Bibliografía}

1. Rosemary Hickey, M.D, Maurice S. Albin, M.D. Autoregulation of Spinal Cord Blood Flow: Is the cord a microcosm of the brain?. Stroke vol 17, no 6, 1986.

2. Melissa C. Werndle; Samira Saadoun; Isaac Phang. Monitoring of Spinal Cord Perfusion Pressure in acute spinal cord injury: Initial findings of the injured spinal cord pressure evaluation study. Critical Care Med 2014; $42: 4$.

3. Soubeyrand Marc, Court Charles, Fadel Elie, Vincent-Mansour César, Mascard Eric, Vanel Daniel, Missenard Gilles. Preoperative imaging study of the spinal cord vascularization: Interest and limits in spine resection for primary tumors. Eur J radiol. 2011; 77: 26-33.

4. Ropper Allan H, Brown Robert H. Principios de Neurología de Adams y Victor; Octava Edición. McGraw-Hill interamericana. 2005; 44: 1067-1068.

5. Ferner Helmut, Staubesand Jochen. Sobotta Atlas de Anatomía Humana. Cap. Médula espinal, nervios raquídeos, tronco simpático. 18 Edición. Medica Panamericana. 1984; 1: 129.

6. Moore Keith, Dalley Arthur. Clinically Oriented Anatomy. 4th Edicion. Médica Panamericana. 2002; 4: 496-497.

7. Tobinick Edward. El sistema venoso cerebroespinal: Anatomía, fisiología e implicaciones clínicas. Medscape General Medicine 8(1): 2006.

8. Serrador JM, Milberg W. And Lipsitz L. Cerebral Hemodynamics in the Elderly From: Current Clinical Neurology Vascular Dementia: Cerebrovascular Mechanisms and Clinical Management Edited by: R. H. Paul, R. Cohen. 2005.

9. C.K. Willie, F.L. Colino, D.M. Bailey, Y.C. Tzeng, Utility of transcranial Doppler ultrasound for the integrative assessment of cerebrovascular function. Journal of Neuroscience Methods 196 (2011) 221-237.

10. Nystrom B, Norton K. Regional spinal cord and brain blood flows in the rat. Neurol Res 5(1): 91-101, 1983.

11. ML Marcus, DD Heistad, JC Ehrhardt and FM Abboud. Regulation of total and regional spinal cord blood flow. Circulation Research. 1977; 41: 128-134.

12. Rosenfeld JV, Maas Al, Bragge $P$, et al: Early management of severe traumatic brain injury. Lancet 2012; 380: 1088-1098.

13. Hadley MN: Blood pressure management after acute spinal cord injury. Neurosurgery 2002; 50: S58-S62.

14. U.K. National Spinal Cord Injury Strategy Board website: The initial management of adults with spinal cord injuries: Advice for major trauma networks and $\mathrm{SCl}$ centres on the development of joint protocols. With advice for clinicians in acute hospitals. Available at: http:// www.excellence.eastmidlands.nhs.uk/welcome/improving-care/emergency urgent-care/major-trauma/major-trauma-related-documents/. Accessed May 1, 2012.

15. Paulson OB, Strandgaard S, Edvinsson L. Cerebral autoregulation, Cerebrovasc Brain Metab Rev. 1990; 2: 161-192.

16. Cottrel James E, Young William L. Cottrel and Young 's Neuroanesthesia. Fifth Edition. Saunders Elsevier. 2010; 2: 17-18.

17. Florence G, Seylaz J. Rapid autoregulation of cerebral blood flow: A laser-Doppler flowmetry study, J Cereb Blood Flow Metab. 1992; 12: 674-680.

18. Sandler AN, Tator CH: Review of the measurement of normal spinal cord blood flow, Brain Res. 1976; 118: 181-198.

19. Shibata K, Takamoto S, Kotsuka Y, et al. Doppler ultrasonographic identification of the critical segmental artery for spinal cord protection, Eur J Cardiothorac Surg. 2001; 20: 527-532.

20. Wagner BP, Ammann RA, Bachmann DC, et al. Rapid assessment of cerebral autoregulation by near-infrared spectroscopy and a single dose of phenylephrine. Pediatr Res 2011; 69(5 pt 1): 436-441.

21. Macnab AJ, Gagnon RE, Gagnon FA. Near infrared spectroscopy for intraoperative monitoring of the spinal cord. Spine 2002; 27: 17-20.

22. Kunihara T, Shiiya N, Matsuzaki K, et al. Near-infrared spectrophotometry is useful to detect the beneficial pharmacological effects of alprostadil on spinal cord deoxygenation. Ann Thorac Cardiovasc Surg 2008; 14: 376-381.

23. Amir Reza Amiri, Cheong Hung Lee, Terence S. Leung. Intraoperative assessment of human spinal cord perfusion using near infrared spectroscopy with indocyanine green tracer technique. The Spine Journal 13 (2013) 1818-1825.

24. Papadopoulos MC, Verkman AS. Aquaporin 4 and neuromyelitis optica. Lancet Neurol 2012; $11: 535-544$

25. Czosnyka M, Guazzo E, Whitehouse M, et al. Significance of intracranial pressure waveform analysis after head injury. Acta Neurochir (Wien) 1996; 138: 531-541.

\section{Correspondencia a:}

José Daniel Charry

Estudiante de Medicina Universidad Surcolombiana.

Hospital Universitario Hernando Moncaleano Perdomo. Neiva, Colombia.

danielcharry06@gmail.com 